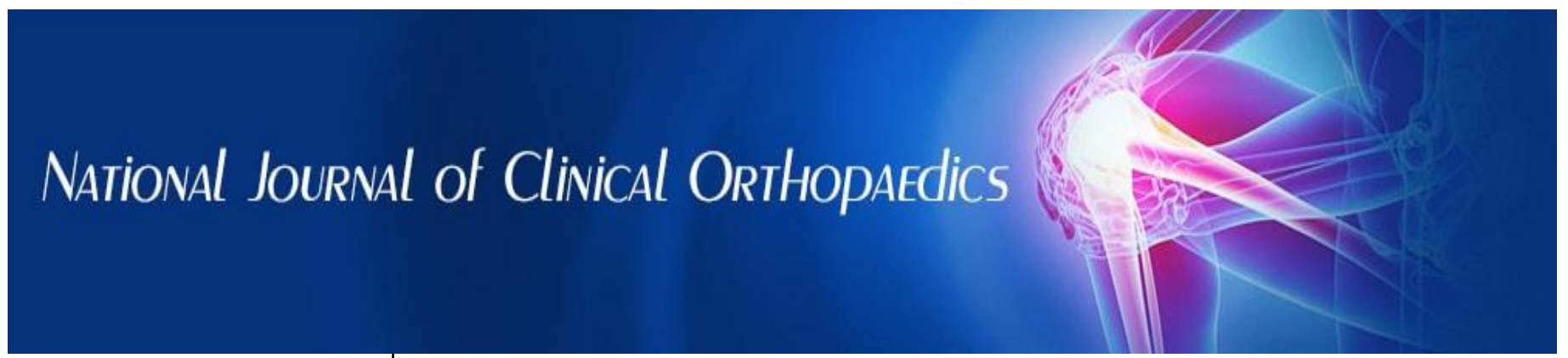

ISSN (P): 2521-3466

ISSN (E): 2521-3474

(C) Clinical Orthopaedics

www.orthoresearchjournal.com

2020; 4(1): 15-18

Received: 09-11-2019

Accepted: 13-12-2019

Dr. Santosh Kumar Sahu

Assistant Professor of

Orthopaedics, IMS\&SUM

Hospital, Bhubaneswar, Odisha,

India

Dr. Sidhartha Samal

Assistant Professor of

Orthopaedics, IMS\&SUM

Hospital, Bhubaneswar, Odisha,

India

Dr. Pushpak Samal

Assistant Professor of

Orthopaedics, IMS\&SUM

Hospital, Bhubaneswar, Odisha,

India

Corresponding Author:

Dr. Santosh Kumar Sahu

Assistant Professor of

Orthopaedics, IMS\&SUM

Hospital, Bhubaneswar, Odisha,

India

\section{Evaluation of risk factors of late Periprosthetic joint infection: A retrospective study}

\author{
Dr. Santosh Kumar Sahu, Dr. Sidhartha Samal and Dr. Pushpak Samal
}

DOI: https://doi.org/10.33545/orthor.2020.v4.i1a.194

\section{Abstract}

Background: Prosthetic Joint Infection that develops at a variable length of time after an index joint replacement procedure is known as LateProsthetic Joint Infection. There must be an asymptomatic period clinical and/or radiographic signs, after an initially successful index procedure. The current study aims to retrospectively identify the risk factors for late PJI following primary total joint arthroplasty and classify those significant risk factors as either non-modifiable or potentially modifiable for intervention prior to surgery. Till date not many studies has been done in Indian subcontinent, which prompted us for the study.

Material and Methods: We report our experience in 33 cases of Late PJI and its causative factors including microorganisms and its subsequent management, of deep infection following over 5000 primary joint replacements carried out over 6 years (2013 may to 2019 September) at IMS \& SUM Hospital, Bhubaneswar. All patients were followed up for delayed PJI for a minimum period of one year postoperatively involving review of all readmissions, returns to the operating room, positive cultures, morbid obesity, UTI, dental procedures, UGI endoscopy, skin diseases etc.

Results: Significant risk factors for late PJI after primary TJA included active tobacco use, diabetes and related comorbidities, S. aureus colonization, UTI, morbid obesity, skin diseases, UGI endoscopy, male gender, age $>=55 \mathrm{yrs}$. These risk factors can be broadly divided into modifiable and non-modifiable risk factors. Modifiable risk factors like such active tobacco use and S. aureus colonization can be eliminated to reduce the incidence of late PJI.

Conclusion: The current study aims to identify the risk factors of late PJI after primary arthoplasty, thus patients can be counselled properly prior to undergoing TJA, which should help them make treatment decisions regarding these procedures. Apart from this the modifiable risk factors can be addressed prior to surgery, thus the overall incidence of late PJI can be reduced.

Keywords: Delayed, peri prosthetic joint infection, primary arthroplasty, risk factors, modifiable, nonmodifiable

\section{Introduction}

Late Periprosthetic joint infections (PJI) following a pimary total joint arthroplasty (TJA) are rare complications but can lead to inferior outcomes. The incidence of late PJI following Total Knee Arthroplasty ranges from $0.5 \%$ to $1.8 \%{ }^{[1,3]}$ and the incidence can be even higher in high-risk groups ${ }^{[4]}$. Late PJI develops at a variable length of time after the pimary arthroplasty procedure. There is an asymptomatic period clinical and/or radiographic signs, after an initially successful index procedure. Late PJI is distinct from infections occurring due to intraoperative contamination. As regards definition of late PJI, most of the literature have suggested that any infection occurring after one year of primary Arthroplasty should be considered as late. Coventry defined 3 stages of PJI. Stage I is an acute infection that occurred within 3 months of the index procedure. Stage II is a delayed infection that occurred between 3 months and 2 years after the index procedure where there was no pain-free interval. Stage III is a hematogenous infection where there is a pain-free stage ${ }^{[5]}$. Garvin and Hanssen defined a late $\mathrm{PJI}$ as one that occurred 4 weeks after the index procedure with an insidious clinical onset ${ }^{[6]}$. McPherson et al. defined a chronic infection as one that had symptoms for 4 weeks or longer [7]. Fares Haddad et al. classified late PJI as late hematogenous PJI, where there was an asymptomatic period followed by clinical and/or radiographical signs of infection. 
The workgroup feels that late PJI arises as a result of bacteraemia at a later stage ${ }^{[8]}$ and should be differentiated from infections occurring due to intraoperative contamination. A number of policies have been adopted perioperatively to minimize the incidence of infection including antibiotic prophylaxis, surgical site preparation, contained surgeon exhaust suits, laminar airflow, and bacterial decolonization of Staphylococcus aureus ${ }^{[9,}{ }^{10]}$. Elimination of modifiable risk factors prior to surgery can reduce the risk of infection following total joint arthroplasty. The aim of the current study is to, retrospectively identify the risk factors for late PJI following primary total joint arthroplasty and to classify them as nonmodifiable or modifiable for intervention prior to surgery. Some of the patient-related factors like diabetes, obesity, S. aureus colonization, and tobacco use have been associated with

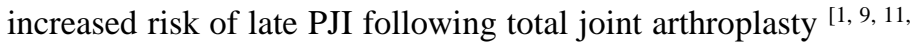
12, 13].

Material and Methods: We analyzed data from 33primary arthroplasty procedures performed at IMS\&SUM Hospital, Bhubaneswar which ended up in delayed PJI. All patients were monitored for delayed PJI for a minimum period of one year postoperatively through an aggressive hospital surveillance program involving review by our infection control department of all readmissions, returns to the operating room, and positive cultures. Prior to surgery, only 16 patients were screened for nasal colonization of $\mathrm{S}$. aureus, prescribed a 5-day course of mupirocin ointment for nasal decolonization, and provided chlorhexidine wipes for application to the skin the evening before and morning of surgery. Among the rest 17 patients who were not screened for nasal colonization of S. aureus, 2 showed growth of S. aureus from wound. Infection prophylaxis during the study timeframe involved administration of intravenous antibiotics within 1 hour prior to incision and continued 24 hours postoperatively. For patients who screened positive for methicillin resistant S. aureus (MRSA), vancomycin was administered as prophylaxis while a second-generation cephalosporin was administered for patients with a negative screen for MRSA. Surgical approach, anaesthesia type, and implant type varied based on patient characteristics and surgeon preference. Postoperative wound management involved sterile dressing for a period of 1-3 days postoperatively with serial dressing changes as needed until the wound was dry. Deep vein thromboembolism (DVT) prophylaxis involved various regimens each of which adhered to the recommendations of the American College of Chest Physicians (ACCP) and the American Academy of Orthopaedic Surgeons (AAOS).Patient demographic and comorbidity data abstracted through the medical chart review are listed in Table 1-7. Diabetes status that involved a positive history of diabetes mellitus and any related organ damage such as retinopathy, neuropathy and nephropathy, was noted. Tobacco use was defined as use within one month prior to surgery. Modifiable risk factors were defined as those factors that could potentially be treated or optimized prior to elective surgery. Questionnaire to study the risk factors subdivided into 3 groups-Hospital related factors, Perioperative factors, Patient related factors.

\section{Analysis}

Table 1: (Total entries)

\begin{tabular}{|c|c|c|}
\hline Total entries & Unilateral & Bilateral \\
\hline TKR & 21 & 3 \\
\hline THR & 5 & 1 \\
\hline TSR & 3 & 0 \\
\hline
\end{tabular}

Table 2: (Sex Distribution)

\begin{tabular}{|c|c|c|c|c|c|c|}
\hline Sex & u/l TKR & b/l TKR & u/l THR & b/l THR & u/l TSR & b/I TSR \\
\hline Male & 15 & 2 & 3 & 1 & 2 & 0 \\
\hline Female & 6 & 1 & 2 & 0 & 1 & 0 \\
\hline
\end{tabular}

Table 3: (Hospital Related Risk Factors)

\begin{tabular}{|c|c|}
\hline Hospital Related Risk Factors & No. of cases attributed \\
\hline Screening for MRSA by nasal \& perineal swab & $3(9.09 \%)$ \\
\hline Catheter associated UTI & $9(27.27 \%)$ \\
\hline Central line associated & 0 \\
\hline
\end{tabular}

Table 4: (Peri-operative Risk Factors)

\begin{tabular}{|c|c|}
\hline Peri-operative Risk Factors & $\begin{array}{c}\text { No. of cases } \\
\text { attributed }\end{array}$ \\
\hline Optimisation of surgical environment & 0 \\
\hline $\begin{array}{c}\text { Prophylactic Antibiotic Administration preparation } \\
\text { of surgical site }\end{array}$ & 0 \\
\hline Contained exhaust suit for surgeon & 0 \\
\hline Laminar airflow OT & 0 \\
\hline Bacterial Decolonization of Staph. aureus & 0 \\
\hline
\end{tabular}

\section{Patient Related Factors}

Table 5: (Non-modifiable Factors)

\begin{tabular}{|c|c|}
\hline Non-modifiable Risk Factors & No. of cases attributed \\
\hline Sex (Male gender) & $23(69.69 \%)$ \\
\hline Age $(>=55 \mathrm{yrs})$ & $24(72.72 \%)$ \\
\hline
\end{tabular}

Table 5: (Disease Related Risk Factors)

\begin{tabular}{|c|c|}
\hline Disease Related Risk Factors & No. of cases attributed \\
\hline Diabetes \& related Comorbidities & $12(36.36 \%)$ \\
\hline Cardiac comorbidities & $3(9.09 \%)$ \\
\hline GI comorbidities & $3(9.09 \%)$ \\
\hline Pulmonary Comorbidities & $1(3.03 \%)$ \\
\hline Skin diseases & $6(18.18 \%)$ \\
\hline Renal comorbidities & $3(9.09 \%)$ \\
\hline Immunocompromised. & $2(6.06 \%)$ \\
\hline
\end{tabular}

Table 6: (Life style Related Risk Factor)

\begin{tabular}{|c|c|}
\hline Life style Related Risk Factor & No. of cases attributed \\
\hline Tobacco use 1month prior to surgery & $3(9.09 \%)$ \\
\hline Morbid obesity & $9(27.27 \%)$ \\
\hline
\end{tabular}

Table 7: (Medical Procedure Related Risk Factors)

\begin{tabular}{|c|c|}
\hline Factors & No. of cases attributed \\
\hline Dental Procedures & $2(6.06 \%)$ \\
\hline UGI endoscopy & $4(12.12 \%)$ \\
\hline Colonoscopy & $3(9.09 \%)$ \\
\hline
\end{tabular}

Table 8: (Organisms Isolated)

\begin{tabular}{|c|c|}
\hline Organisms Isolated & No. of cases \\
\hline Klebsiella & $5(15.15 \%)$ \\
\hline Enterococci & $3(9.09 \%)$ \\
\hline Acinetobacter & $5(15.15 \%)$ \\
\hline Enterobacter cloacae & $3(9.09 \%)$ \\
\hline Staph. aureus & $3(9.09 \%)$ \\
\hline E. coli & $12(36.36 \%)$ \\
\hline Pseudomonas & $2(6.06 \%)$ \\
\hline
\end{tabular}


Table 9: (Management options)

\begin{tabular}{|c|c|}
\hline Management options & No. of cases \\
\hline Debridement only & $3(9.09 \%)$ \\
\hline Debridement with insert exchange & $10(30.30 \%)$ \\
\hline 2 stage revision & $18(54.54 \%)$ \\
\hline Arthrodesis & $2(6.06 \%)$ \\
\hline
\end{tabular}

\section{Discussion}

Late PJI following primary arthroplasty can lead to inferior outcomes and increased costs. The current study aims to identify risk factors of late PJI following primary arthroplasty and to classify them as non-modifiable or modifiable by intervention prior to surgery. Significant risk factors for infection after primary TKA included active tobacco use, DM and S. aureus colonization. Some of these risk factors such as tobacco use and S. aureus colonization are potentially modifiable, and efforts to minimize or eliminate these modifiable risk factors may help to improve late PJI rates following primary TJA. Several studies have suggested association between $\mathrm{S}$. aureus nasal colonization and development of PJI $[12,14,15] .18-24 \%$ of the general population I have been shown to be colonized with S. aureus [16, ${ }^{17]}$. Surgical site infection (SSI) involving S. aureus is associated with preoperative nasal colonization ${ }^{[18]}$. In our study S. aureus colonization is a significant and independent risk factor for late PJI following primary arthroplast, with a total of 3 cases $(9.09 \%)$ out of 33 cases. Tobacco use among our patients within one month prior to surgery was an independent and significant predictor of risk for infection following primary arthroplasty, with a total of-cases. Singh et al. suggested that active tobacco use had a significantly increased risk compared to those with no prior tobacco use ${ }^{[19]}$. In our study we found that active tobacco use had additive effects with other risk factors. Uncontrolled diabetes is usually associated with increased risk of infection and poor wound healing ${ }^{[9]}$. Marchant et al. found uncontrolled diabetes can increase infection rates by two-fold ${ }^{[20]}$. In the our study population, a diagnosis of diabetes with related complications were found to be a significant and independent risk factor for late PJI, with a total of 12 cases $(36.36 \%)$. 9cases had morbid obesity $(27.27 \%)$. Medical procedures like, dental procedures, GI endoscopy prior to primary arthroplasty can cause bacteraemia and has been attributed as potential risk factor for PJI. In our study population, 4 patients (12.12\%) had undergone uppe GI endoscopy, 2 cases (6.06\%) dental procedures and $3(9.09 \%)$ cases had undergone colonoscopy. In our study E. coli was the most common organism isolated, with 12 cases $(36.36 \%)$ showing growth of the organism, followed by Klebsiella and Acinetobacter 5cases each (15.15\%). 18cases $(54.54 \%)$ were managed by two stage revision surgery, where stage-I involved, removal of implants, thorough debridement, antibiotic spacer placement and administration of IV antibiotics as per the culture and sensitivity reports. Stage-II involved removal of spacer and application of revision implants, once parameters like ESR, CRP became normal following application of antibiotic spacer and IV antibiotic administration. 10 cases $(30.30 \%)$ were managed with thorough debridement, insert exchange and administration of IV antibiotics. 3 cases (9.09\%) with superficial infections were managed with thorough debridement and antibiotics. 2 cases $(6.06 \%)$ with failed revision surgeries due to recurrent infections, were managed by arthrodesis of the joint involved along with IV antibiotics.

\section{Conclusion}

The current study aimed to identify the risk factors of late PJI after primary arthroplasty, thus patients can be counselled properly prior to undergoing total Joint arthroplasty, which should help them make treatment decisions regarding these procedures. Apart from this the modifiable risk factors can be addressed prior to surgery, thus the overall incidence of late PJI can be reduced.

\section{References}

1. Namba RS, Inacio MC, Paxton EW. Risk factors associated with deep surgical site infections after primary total knee arthroplasty: an analysis of 56,216 knees. JBJS. 2013; 95(9):775.

2. Kurtz SM, Lau E, Schmier J et al. Infection burden for hip and knee arthroplasty in the United States. J Arthroplasty. 2008; 23(7):984.

3. Pruzansky JS, Bronson MJ, Grelsamer RP et al. Prevalence of modifiable surgical site infection risk factors in hip an knee joint arthroplasty patients at an urban academic hospital. J Arthroplasty. 2014; 29:272.

4. Chesney D, Sales J, Elton R, et al. Infection after knee arthroplasty: a prospective study of 1509 cases. J Arthroplasty. 2008; 23(3):355.

5. Coventry MB. Treatment of infections occurring in total hip surgery. Orthop Clin North Am. 1975; 6(4):991.

6. Garvin KL, Hanssen AD. Infection after total hip arthroplasty. Past, present, and future. J Bone Joint Surg Am. 1995; 77(10):1576.

7. McPherson EJ, Woodson C, Holtom $\mathrm{P}$ et al. Periprosthetic total hip infection: outcomes using a staging system. Clin Orthop Relat Res. 2002; 403:8

8. Sendi P, Banderet F, Graber P et al. Clinical comparison between exogenous and haematogenous periprosthetic joint infections caused by Staphylococcus aureus. Clin Microbiol Infect. 2011; 17(7):1098.

9. Illingworth KD, Mihalko WM, Parvizi J et al. How to minimize infection and thereby maximize patient outcomes in total joint arthroplasty: a multicentre approach: AAOS exhibit selection. JBJS. 2013; 95(8):e50.

10. Bosco J, Slover J, Haas J. Perioperative strategies for decreasing infection. JBJS. 2010; 92-A(1):231.

11. Bosco J, Slover J, Haas J. Perioperative strategies for decreasing infection. JBJS. 2010; 92-A(1):231.

12. Jamsen E, Furnes O, Engesaeter LB et al. Prevention of deep infection in joint replacement surgery. Acta Orthop. 2010; 81(6):660.

13. Maoz G, Phillips M, Bosco J et al. Modifiable vs. nonmodifiable risk factors for infection after hip arthroplasty [abstract]. Presented at: Twentieth Combined Open Meeting of the Hip Society And The American Association Of Hip And Knee Surgeons (AAHKS); March 2014; New Orleans, Louisiana, 2014.

14. Wenzel RP, Perl TM. The significance of nasal carriage of Staphylococcus aureus and the incidence of postoperative wound infection. J Hosp Infect. 1995; 31(1):13.

15. Skramm I, Fossum Moen AE, Aroen A et al. Surgical site infections in orthopaedic surgery demonstrate clones similar to those in orthopaedic Staphylococcus aureus nasal carriers. JBJS. 2014; 96(11):882.

16. Wertheim HF, Vos MC, Ott A et al. Risk and outcome of nosocomial Staphylococcus aureus bacteraemia in nasal carriers versus non-carriers. Lancet. 2004; 364(9435):703.

17. Bode LG, Kluytmans JA, Wertheim HF et al. Preventing surgical-site infections in nasal carriers of Staphylococcus aureus. N Engl J Med. 2010; 362(1):9.

18. Mangram AJ, Horan TC, Pearson ML et al. Guideline for 
prevention of surgical site infection, 1999. Hospital Infection Control Practices Advisory Committee. Infect Control Hosp Epidemiol. 1999; 20(4):250.

19. Singh JA, Houston TK, Ponce BA et al. Smoking as a risk factor for short-termoutcomes following primary total hip and total knee replacement in veterans. Arthritis Care Res (Hoboken). 2011; 63(10):1365.

20. Marchant Jr MH, Viens NA, Cook C et al. The impact of glycemic control and diabetes mellitus on perioperative outcomes after total joint arthroplasty. JBJS. 2009; 91(7):1621. 